\title{
BMJ Open Protocol for a multisite randomised trial of Hand-Arm Bimanual Intensive Training Including Lower Extremity training for children with bilateral cerebral palsy: HABIT-ILE Australia
}

\author{
Leanne Sakzewski, ${ }^{1}$ Yannick Bleyenheuft, ${ }^{2}$ Roslyn N Boyd, ${ }^{1}$ Iona Novak, ${ }^{3}$ \\ Catherine Elliott, ${ }^{4}$ Sarah Reedman, ${ }^{1}$ Cathy Morgan, ${ }^{3}$ Kerstin Pannek, ${ }^{5}$ \\ Jurgen Fripp, ${ }^{5}$ Prue Golland, ${ }^{3}$ David Rowell, ${ }^{6}$ Mark Chatfield, ${ }^{1}$ Robert Stuart Ware ${ }^{7}$
}

To cite: Sakzewski L, Bleyenheuft Y, Boyd RN, et al. Protocol for a multisite randomised trial of Hand-Arm Bimanual Intensive Training Including Lower Extremity training for children with bilateral cerebral palsy: HABIT-ILE Australia. BMJ Open 2019;9:e032194. doi:10.1136/ bmjopen-2019-032194

- Prepublication history for this paper is available online To view these files, please visit the journal online (http://dx.doi. org/10.1136/bmjopen-2019032194).

Received 10 June 2019 Revised 17 July 2019 Accepted 18 July 2019

Check for updates

(C) Author(s) (or their employer(s)) 2019. Re-use permitted under CC BY-NC. No commercial re-use. See rights and permissions. Published by BMJ.

For numbered affiliations see end of article.

Correspondence to Dr Leanne Sakzewski; I.sakzewski1@uq.edu.au

\section{ABSTRACT}

Introduction Children with bilateral cerebral palsy often experience difficulties with posture, gross motor function and manual ability, impacting independence in daily life activities, participation and quality of life (QOL). Hand-Arm Bimanual Intensive Training Including Lower Extremity (HABIT-ILE) is a novel intensive motor intervention integrating upper and lower extremity training. This study aimed to compare HABIT-ILE to usual care in a large randomised controlled trial (RCT) in terms of gross motor function, manual ability, goal attainment, walking endurance, mobility, self-care and QOL. A within-trial cost-utility analysis will be conducted to synthesise costs and benefits of HABIT-ILE compared with usual care.

Methods and analysis 126 children with bilateral cerebral palsy aged 6-16 years will be recruited across three sites in Australia. Children will be stratified by site and Gross Motor Function Classification System and randomised using concealed allocation to either receiving HABIT-ILE immediately or being waitlisted for 26 weeks. HABIT-ILE will be delivered in groups of 8-12 children, for 6.5 hours per day for 10 days (total 65 hours, 2 weeks). Outcomes will be assessed at baseline, immediately following intervention, and then retention of effects will be tested at 26 weeks. Primary outcomes will be the Gross Motor Function Measure and ABILHAND-Kids. Secondary outcomes will be brain structural integrity, walking endurance, bimanual hand performance, self-care, mobility, performance and satisfaction with individualised goals, and QOL. Analyses will follow standard principles for RCTs using two-group comparisons on all participants on an intention-to-treat basis. Comparisons between groups for primary and secondary outcomes will be conducted using regression models.

Ethics and dissemination Ethics approval has been granted by the Medical Research Ethics Committee of Children's Health Queensland Hospital and the Health Service Human Research Ethics Committee (HREC/17/ QRCH/282) of The University of Queensland (2018000017/ HREC/17/QRCH/2820), and The Cerebral Palsy Alliance Ethics Committee (2018_04_01/HREC/17/QRCH/282).

Trial registration number ACTRN12618000164291.

\section{Strengths and limitations of this study}

- This is a large randomised controlled trial investigating the efficacy of an intensive motor training approach to improve gross motor function and manual ability for children with bilateral cerebral palsy, powered to test both primary and secondary outcomes.

- Potential participants will be recruited from three centres in Australia, ensuring that the sample size will be met.

- Outcomes include gross motor function, manual ability, brain structure and function, self-care, mobility, bimanual performance, quality of life, and self-perceived performance of and satisfaction with individually defined functional goals.

- A fidelity framework includes standardised training of interventionists and fidelity monitoring of each intervention day camp.

- A comprehensive within-trial cost-utility analysis will be conducted to synthesise the costs and benefits of the Hand-Arm Bimanual Intensive Training Including Lower Extremity programme compared with usual care.

\section{INTRODUCTION}

Cerebral palsy (CP) is the most common physical disability in childhood ${ }^{1}$ with an estimated prevalence of 1.4 in 1000 live births. ${ }^{2}$ Six hundred children are newly diagnosed with CP each year, with greater than 35000 people living with CP in Australia. ${ }^{3}$ Over $61 \%$ of children with CP have 'bilateral' motor involvement, impairing movement on both sides of the body. ${ }^{1}$ For some of these children, all four limbs and trunk are affected, making both walking and effective upper limb use challenging. These limitations significantly impact their independence and participation in home, school, work and community life. ${ }^{4}$ People with CP have poorer health outcomes compared 
with age-matched peers. ${ }^{5}$ Increased severity of physical disability is associated with reduced general health, greater pain and discomfort, ${ }^{5}$ reduced independence in daily life skills $^{6}$ and poorer vocational outcomes. ${ }^{7}$ Interventions that reduce the impact of the physical disability and promote independence in daily life skills, inclusion and community participation are essential.

Traditional neurodevelopmental interventions were frequently based on passive movement experiences using passively guided movements (with the aim of normalising movement), as well as passive manual stretching (aimed to improve or maintain range of motion and to decrease contractures and spasticity). These have been shown to be ineffective in improving motor outcomes for children with $\mathrm{CP}^{8}{ }^{9}$ Contemporary and proven effective interventions for school-aged children with CP involve child-active task-specific motor training from the motor learning paradigm, such as constraint-induced movement therapy, bimanual training and goal-directed training. ${ }^{8}{ }^{9}$ Since these interventions predominantly target upper and lower extremity motor performance separately, the evidence bases are different. ${ }^{8}$ There have been fewer studies investigating task-specific interventions to target lower compared with upper limb motor performance. A recent systematic review identified the effectiveness of specific gait training in increasing gait speed for children with unilateral and bilateral CPs (effect size $=0.92, p=0.01$ ) ${ }^{10}$ To date, significant evidence exists for intensive upper extremity interventions $(\approx 60$ hours) to enhance upper limb motor performance in children with unilateral CP. ${ }^{8}$ A number of systematic reviews and meta-analyses ${ }^{8911}$ have confirmed growing evidence for intensive contemporary motor learning-based approaches to upper limb training for school-aged children with unilateral CP (eg, constraint-induced movement therapy, HandArm Bimanual Intensive Training) to improve upper limb motor performance.

Since children with bilateral CP often have both upper and lower limbs involved, Hand-Arm Bimanual Intensive Training Including Lower Extremity (HABIT-ILE) was invented to treat both the upper and lower limbs concurrently. In children with unilateral $\mathrm{CP}$, a randomised control trial (RCT) demonstrated the efficacy of HABIT-ILE for both the upper and lower extremities. ${ }^{12}$ Results obtained from concurrent upper and lower extremity training were similar to those obtained by children who underwent upper extremity training alone. ${ }^{13}$ These findings led researchers to test whether HABIT-ILE intervention might be helpful for children with bilateral CP. ${ }^{14}$ A recent systematic review of interventions to improve upper limb function in children with bilateral CP, however, has found a large variety of different interventions addressing upper limb function, but most studies have weak to moderate methodological quality. ${ }^{15}$ The strongest evidence was from a small quasi-RCT of HABIT-ILE, and the authors highlighted the need for further high-quality trials. ${ }^{14}$ Compared with a waitlist control group, children with bilateral CP who underwent 84hours of HABIT-ILE achieved significantly greater gains in manual ability (ABILHAND-Kids $\mathrm{n}^{2}=0.32$, $\mathrm{p}<0.001)$, self-care on the Paediatric Evaluation of Disability Inventory $\left(\mathrm{n}^{2}=0.26, \mathrm{p}=0.001\right)$, gross motor function on the Gross Motor Function Measure 66-item (GMFM-66: $\mathrm{n}^{2}=0.33, \mathrm{p}<0.001$ ), walking distance on the 6 min Walk Test (6MWT: $\mathrm{n}^{2}=0.17, \mathrm{p}<0.03$ ) and balance on the Paediatric Balance Scale $\left(\mathrm{n}^{2}=0.28, \mathrm{p}<0.002\right)$. These promising results indicate that a larger RCT is warranted to confirm the efficacy of HABIT-ILE on manual ability and gross motor function for children with bilateral CP. This multisite RCT, HABIT-ILE Australia, will compare this intensive motor training approach to usual care in school-aged children with bilateral $\mathrm{CP}$ at a lower dose than the original study (65 hours vs 90 hours). This lower dose was selected based on potential acceptability and feasibility within the Australian context.

\section{AIMS AND HYPOTHESES \\ Broad aim}

This multisite RCT will be conducted in three Australian states (Queensland (QLD), New South Wales (NSW) and Western Australia (WA)) with 126 school-aged children with bilateral CP. This RCT with a pragmatic, single-blind design will determine if HABIT-ILE is more effective than usual care to improve manual ability (ABILHAND-Kids) and gross motor function (GMFM-66) immediately postintervention and retention at 26 weeks. Secondary outcomes will test the differential effects of HABIT-ILE compared with usual care on neuroplastic changes in brain structural integrity, functional and structural connectivity, walking endurance (6MWT), self-care and mobility (Paediatric Evaluation of Disability Inventory-Computerised Assessment Test (PEDI-CAT)), bimanual performance (Both Hands Assessment: BoHA), performance of and satisfaction with individualised occupational performance goals (Canadian Occupational Performance Measure (COPM)), and quality of life (QOL) (Cerebral Palsy Quality of Life (CP QOL) Questionnaire: CP QOL-Child or CP QOL-Teen) immediately postintervention and retention at 26 weeks after the intervention.

\section{Primary hypotheses}

For children with bilateral CP, HABIT-ILE for a duration of 65 hours will be more effective than a waitlist control group receiving usual care to improve

1. Manual ability on the ABILHAND-Kids by a difference of 1.6 logits or greater.

2. Gross motor function on the GMFM-66 by a difference of 5 points or greater.

\section{Secondary hypotheses}

For children with bilateral CP, HABIT-ILE for a duration of 65 hours will be more effective than a waitlist control group receiving usual care to increase

1. Brain structural integrity measured using functional MRI (fMRI)-guided tractography. ${ }^{16}$

2. Walking endurance (6MWT).${ }^{17}$ 


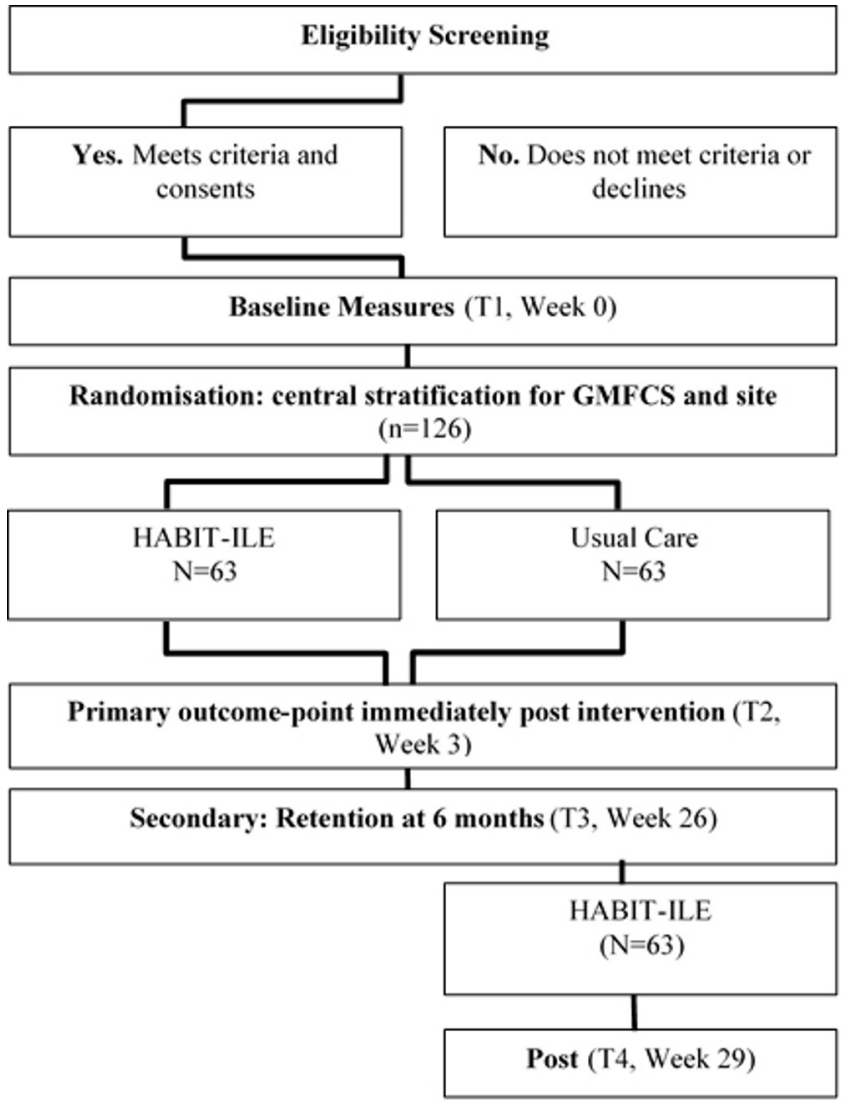

Figure 1 Participant flow diagram for HABIT-ILE Australia. GMFCS, Gross Motor Function Classification System; HABITILE, Hand-Arm Bimanual Intensive Training Including Lower Extremity.

3. Bimanual hand performance (Both Hands Assessment (BoHA) $)^{18}$

4. Self-care and mobility (PEDI-CAT). ${ }^{19}$

5. Performance and satisfaction scores on the COPM. ${ }^{20}$

6. QOL (CP QOL-Child or CP QOL-Teen, parent proxy and child report; and the Child Health Utility Index (CHU9) parent proxy). ${ }^{21} 22$

7. Cost effectiveness ( $\triangle \$$ Cost $/ \triangle C P Q O L)$ of medical treatment received.

\section{METHODS}

\section{Study design}

This single-blind RCT will compare HABIT-ILE to usual care for school-aged children with bilateral CP. Study design has been informed by ConsolidatedStandards of Reporting Trials Guidelines (see figure 1).

\section{Recruitment}

One hundred twenty-six school-aged children between 6 and 16 years of age at study entry with bilateral CP will be recruited. Families with a child meeting eligibility criteria will be invited to join the study through our three collaborating sites and associated clinical services (Queensland Children's Hospital, Cerebral Palsy Alliance and Perth Children's Hospital). Recruitment from three major metropolitan centres will enable the target sample size to be achieved (50 in NSW, 50 in QLD and 26 in WA).

Recruitment at each site will begin following ethical and governance approvals. Recruitment will draw on current databases within each organisation, referrals from clinical services. Based on population numbers available on the Australian Cerebral Palsy Register (1240 potentially eligible participants) and well-established state-wide clinical networks, recruitment of 126 participants is feasible across the three sites. It is expected that final data collection will occur in July 2021.

\section{Inclusion criteria}

To be eligible for inclusion, participants must be

1. Diagnosed with bilateral CP (diplegia/triplegia/ quadriplegia),

2. Gross Motor Function Classification System (GMFCS) levels II (walks with limitations) to IV (limited self-mobility but able to do a standing transfer with the assistance of one person).

3. Aged 6-16 years.

4. Able to grasp light objects and lift most impaired arm $\geq 15 \mathrm{~cm}$ above a table surface.

5. Able to understand instructions and complete testing.

\section{Exclusion criteria}

1. Uncontrolled seizures.

2. Had orthopaedic and/or neurological surgery in the 6 months prior to or scheduled during study period (eligible for inclusion if at least 6 months postsurgery, and/or returned to presurgical gross motor and upper limb function following selective dorsal rhizotomy and no longer undergoing postoperative rehabilitation).

3. A visual impairment interfering with treatment/testing.

4. Inability to undertake standing transfers and/or walk a few steps (with a walker).

5. A significant cognitive and/or behavioural impairment limiting the ability to follow instructions determined through discussions with the primary caregiver and/or during a screening assessment.

6. Non-English speaking.

\section{Randomisation}

A biostatistician will create one central randomisation schedule using computer-based random numbers (in blocks of various sizes ranging from 10 to 20) to receive HABIT-ILE immediately or to waitlist usual care. Children will be stratified based on site (QLD, NSW and WA) and GMFCS (II vs III-IV). After consent and baseline measures are completed, children will be randomised with the use of a REDCap randomisation module set up by non-study personnel.

\section{Blinding}

At all time points, the GMFM-66 and BoHA will be rated from videos ${ }^{23}$ by a certified rater masked to both group allocation and timing of assessments. Parents and assessing clinicians will be masked to group allocation for baseline 
Table 1 TIDieR checklist ${ }^{22}$ : comparison between HABIT-ILE and traditional 'usual care' intervention

\begin{tabular}{ll}
\hline Item & Experimental HABIT-ILE \\
\hline Name & HABIT-ILE \\
Why & Rationale: Intense, repetitive, active motor learning induces \\
activity-dependent neuroplasticity. & Essential elements: \\
& 1. Goal directed (goals defined by child/caregiver). \\
2. $\quad$ Motor training with concurrent challenge for upper and \\
3. Sher limbs and posture. \\
4. Active practice of goals. \\
5. High repetition and intensity.
\end{tabular}

\section{Control traditional usual care}

Traditional eclectic usual care.

Rationale: Usual care is highly

variable, based on biomechanical and neurodevelopmental principles.

Elements may include

1. Goals defined either by child/caregiver or therapist.

2. Stretching, splinting and casting.

3. Strengthening.

4. Functional training (eg, multimodal joint movements).

5. Therapist physically facilitates more typical (normal) movement patterns with children who are passive recipients.

6. May involve active goal practice.

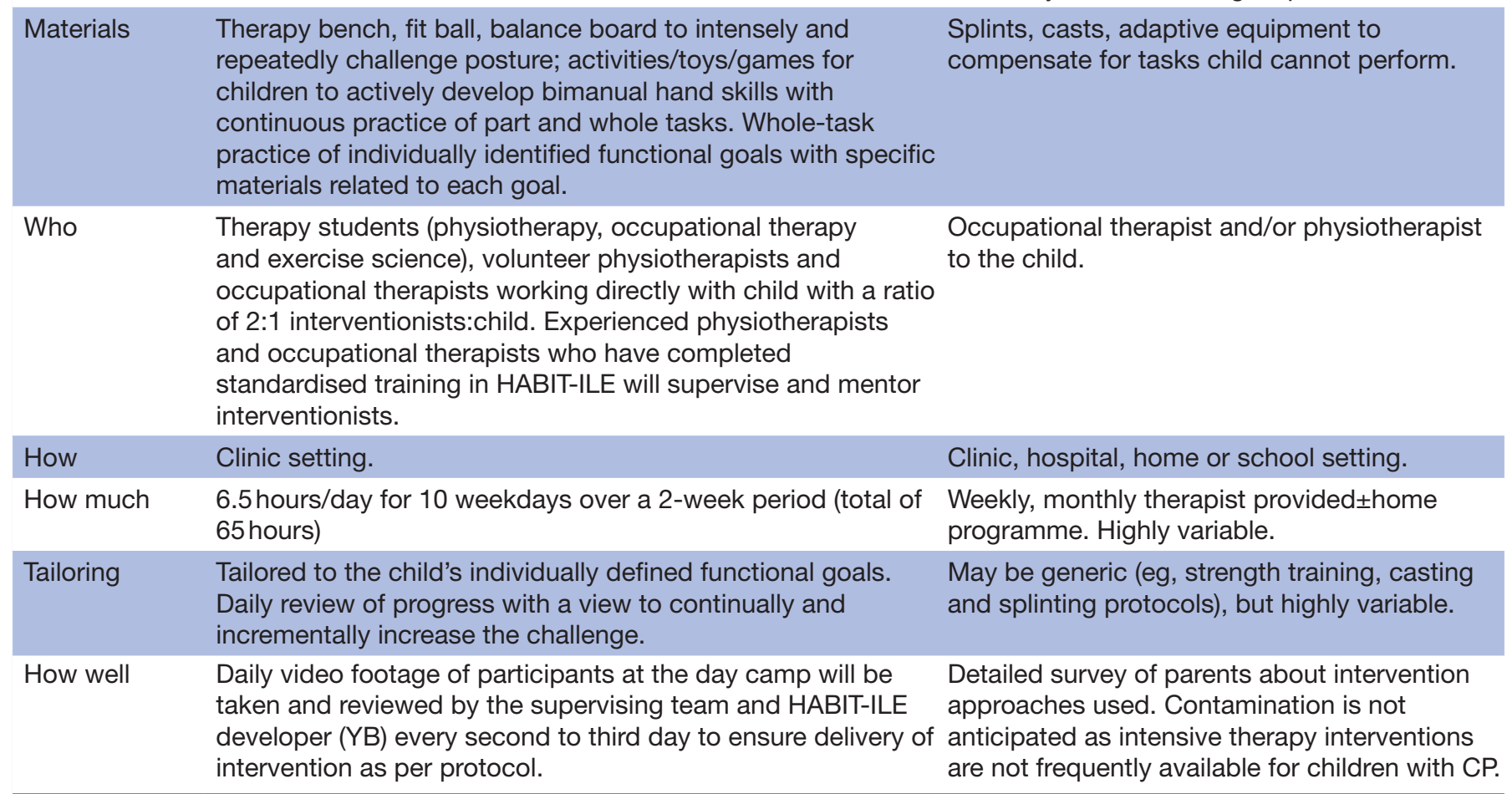

CP, cerebral palsy ; HABIT-ILE, Hand-Arm Bimanual Intensive Training Including Lower Extremity; TIDieR, Template for Intervention Description and Replication.

assessments. Analyses will be conducted using coded group allocation.

\section{Study interventions}

The HABIT-ILE and control interventions are summarised according to the Template for Intervention Description and Replication (TIDieR) Checklist ${ }^{24}$ in table 1.

1. HABIT-ILE is a motor learning approach simultaneously addressing coordination of the upper and lower limbs. ${ }^{12}$ Key elements of HABIT-ILE:

Dose: The total dose is 65 hours of HABIT-ILE. The 65 hours will be achieved through a 2-week intensive group-delivered day camp for 6.5 hours/day over 10 days conducted during the school holidays. Results from our previous research in intensive upper limb training in unilateral $\mathrm{CP}^{25-27}$ and from our systematic review of all upper limb interventions ${ }^{8}$ indicate that 60 hours is likely to be a sufficient dose to achieve significant changes in motor performance, and the 2-week camps are feasible for children and their families. The model of HABIT-ILE to be tested has been adapted to maximise future clinical translation to ensure acceptability and feasibility to children with bilateral CP and their families in Australia.

Mode: Groups of 8-12 children (1:1 or 2:1 therapist/ volunteer/student to child ratio according to ability).

Content and tailoring: Intervention will be based on the child's motor abilities (determined at baseline), age, interests and self-identified functional goals. Tasks/activities are 
made incrementally more challenging. Practice is structured, using part-task and whole-task practice with high repetition and ongoing feedback about performance. ${ }^{12} 28$ A process of shaping with progressive approximations is undertaken over 10 days. For example, if a pincer grasp is required for the goal (eg, do buttons up on school shirt) and the child is not yet performing this grasp effectively, children will practice tasks that progress incrementally towards this grasp. This requires a clinical reasoning process whereby components/impairments leading to the breakdown of goal performance are identified (eg, strength, active range of motion, coordination, and motor planning or strategy) then targeted with deliberately selected, incrementally challenging games and activities.

Upper extremity: Tasks that will be performed include (1) incremented table top fine motor activities, (2) activities of daily living when sitting/standing/walking, and (3) gross motor play and physical activities.

Lower extremity and postural control: Based on the child's baseline motor abilities, postural control/sitting balance will be targeted by sitting on a bench (without postural supports), sitting on an inflated fitness ball, standing with/ without upper limb support and/or standing on a balance board. Postural control will be incrementally challenged by increase of duration of sitting or standing, by increasing of inflation of the fitness balls, progression from one mode to a more difficult one (eg, standing on a flat hard surface to standing on a balance board) and/or introduction of physical and task demands. Children will also engage in gross motor part and whole practice relevant to their functional goals. These may include transfers (sit to stand or floor to stand), stair climbing, walking, running and/or other physical activities.

Intervention providers: A minimum of one physiotherapist and one occupational therapist delivering HABIT-ILE at each site will complete standardised training provided by the developer of HABIT-ILE (YB). This will coincide with the first intensive intervention camp conducted in Brisbane, Australia. The trained therapists will, in turn, train and supervise therapy students, volunteers and therapists to deliver HABIT-ILE in the subsequent camps at their site.

Location: The intervention groups will be conducted in the clinics in each of the participating sites.

2. Usual care: Usual care over the 6-month waitlist period will vary for children with CP across Australia and can range from weekly clinic-based therapy sessions to school-based consultative services provided on a monthly, quarterly or yearly basis. In order to understand the variability in usual care received, all families in both groups will complete a health resource use questionnaire at baseline and 6 months postintervention. This will capture the duration of physiotherapy, occupational therapy and any other concurrent medical interventions, such as intramuscular botulinum toxin A injections and/or serial casting. All children in the usual care group will be offered HABIT-ILE commencing at the subsequent school holiday following 6 months' retention time point (T3).

\section{Adverse events and safety}

Any minor or major adverse event associated with HABIT-ILE will be screened on a daily basis by the treating therapist by verbal questioning and will inform the study coordinator and chief investigators (except major adverse events or those requiring medical treatment, which must be reported as soon as possible, and within 24 hours). Minor adverse events include

- Near miss accidents (such as falling off a bike or falling heavily in a game).

- Sore muscles, bruises and other minor injuries not requiring medical treatment.

- Feeling upset, guilty or sad.

Major adverse events include

- Injuries that require medical treatment (such as moderate-severe strains or broken bones).

After reporting to the site chief investigator, local site processes will be followed as necessary.

\section{Fidelity}

\section{Therapist attributes}

It is required that HABIT-ILE therapists at each site possess the following attributes:

- Full registration with the Australian Health Practitioner Regulation Agency (physiotherapists and occupational therapists).

- Current basic first aid and cardiac pulmonary resuscitation certificate.

It is highly desirable that therapists possess the following attributes:

- Three or more years of experience working with children with $\mathrm{CP}$ and their families.

- Experience working within models or frameworks of motor learning.

\section{Therapist training}

Standardised therapist training will be provided to the core group of therapists (a minimum of one physiotherapist and one occupational therapist from each site) employed to deliver the HABIT-ILE intervention across the three sites. The training package will include

- Intervention manual/resources.

- Onsite training during the first HABIT-ILE camp led by the HABIT-ILE developer (YB).

Training sessions will be video-recorded and accessible at any time for established or new therapists delivering the intervention. In subsequent camps, the trained therapists at each site will deliver the 1-day training to local site staff and students prior to the commencement of each camp.

\section{Fidelity monitoring}

Video footage will be taken for each participating child of the training and progress of tasks towards goal attainment every day/second day during each HABIT-ILE camp. Video footage will be reviewed by the HABIT-ILE developer (YB), with regular meetings scheduled throughout each camp to provide feedback on the intensity of delivery, and ongoing support and recommendations for treating therapists. 


\section{Screening and descriptive measures}

All participants will be classified using the

1. Manual Abilities Classification System (MACS): The MACS will classify the child's ability to hand objects in daily activities on a five-level ordinal scale. ${ }^{29}$ The MACS has established construct validity and excellent inter-rater reliability (intraclass correlation coefficient (ICC) 0.97 between therapists). It is expected that children in the study will be functioning at MACS levels I-III. $^{29}$

2. GMFCS Expanded and Revised: The GMFCS classifies the child's ability to carry out self-generated movements related to sitting and walking on a five-level ordinal scale. ${ }^{30}$ The GMFCS has established construct validity and good inter-rater reliability between therapists. ${ }^{31}$

3. Communication Function Classification System (CFCS): The CFCS will be used to classify children's everyday performance of communicating using all methods (eg, speech, gestures, eye gaze, augmentative and alternative communication) on a five-level ordinal scale. ${ }^{32}$ There is evidence of content validity, good testretest reliability and good inter-rater reliability (0.66) between professionals. $^{32} 33$

Demographic Questionnaire: A study-specific demographic questionnaire will collect information on the child's age, gender, comorbidities, type of schooling, socioeconomic status, family structure and support, family income, and current involvement in rehabilitation programme.

\section{Primary outcomes}

1. ABILHAND-Kids is a Rasch-built parent completed questionnaire measuring the manual ability of children with $\mathrm{CP}^{34}$ The ABILHAND-Kids has demonstrated content, construct and evaluative validity, high internal consistency $(\alpha=0.94)$, excellent test-retest reliability $(r=0.91)^{34}$ and is responsive in detecting change following intensive upper limb motor training interventions (smallest detectible difference $=0.81-1.03$ logits). ${ }^{35} 36$ The ABILHANDS has the strongest evidence of validity and reliability to measure hand function in children with bilateral $\mathrm{CP}^{37}$ and is responsive to change. ${ }^{35}$

2. The GMFM-66 is a criterion referenced observation measure developed using Rasch modelling to measure gross motor function of children with $\mathrm{CP} .{ }^{38}$ The GMFM66 has established construct validity, high test-retest reliability (ICC 0.99$)^{38}$ and is responsive to change (minimal clinically important difference $=1.5) .^{38-40}$

\section{Secondary outcomes}

1. Brain structural integrity: Brain MRI will be conducted using 3T scanners. The child will be familiarised with the MRI procedures before the scan. During the MRI, the child will watch an age-appropriate movie of his or her choice, except during the acquisition of the fMRI. Structural brain images will be acquired using high-resolution 3D T1-weighted magnetization-prepared rapid acquisition with gradient echo (MPRAGE) and high-resolution 3D T2-weighted fluid-attenuated inversion recovery (FLAIR). Diffusion MRI data will be acquired using a multishell approach with 20 directions at $\mathrm{b}=1000 \mathrm{~s} / \mathrm{mm}^{2}, 60$ directions at $\mathrm{b}=3000 \mathrm{~s} / \mathrm{mm}^{2}$ and 8 non-diffusion-weighted images $\left(b=0 \mathrm{~s} / \mathrm{mm}^{2}\right)$. The acquisition will be split in four blocks (of 22 directions) to allow more efficient rescanning of data affected by motion. Half of the blocks are reverse phase encoded to assist in the correction of residual distortions due to susceptibility inhomogeneities. fMRI data will be acquired using a block design, with a simple active hand and passive foot-tapping task. Two two-dimensional gradient recalled echo images (echo timeTE1/ TE2, 4.92/7.38ms) were used to acquire a field map for functional data, which assists when correcting for distortion due to susceptibility inhomogeneities. The total scan time will be $<1$ hour.

Structural brain images will be used for lesion scoring using the Fiori scale, a semiquantitative scale for use in brain imaging of $\mathrm{CP}{ }^{41}$ Structural brain images will also be used to assess alterations in cortical thickness in response to therapy. Diffusion data will allow both traditional analysis using the diffusion tensor model (fractional anisotropy and mean diffusivity), as well as state-of-the-art tractography and calculation of advanced imaging microstructural biomarkers thought to closely reflect the status of the underlying brain tissue. fMRI-guided tractography will be carried out as described previously. ${ }^{42} 43$

2. Walking endurance: The $6 \mathrm{MWT}$ is a clinical exercise test measuring walking endurance with excellent test-retest reliability (ICC 0.98) for children with CP. ${ }^{17}$ The test requires participants to walk as far as possible in 6 min using a $10 \mathrm{~m}$ track with cones demarcating the turning points. Participants will be given verbal and visual instructions before testing. Participants will be instructed to walk as far as possible without running in 6 min. Participants will be given verbal encouragement and will be advised every $30 \mathrm{~s}$ of the distance covered (in laps) and the time remaining. Distance will be measured to the nearest $1 \mathrm{~m}$ mark.

3. Bimanual hand performance: The BoHA measures how children who have bilateral CP use their hands together in bimanual activities. ${ }^{18}$ The measure was developed through adaptation of the Assisting Hand Assessment. Rasch measurement modelling showed strong evidence of internal construct validity, with two separate item difficulty hierarchies for children with (1) symmetric upper limb use and (2) asymmetric upper limb use. ${ }^{17}$ The test uses a selection of toys to elicit bimanual hand behaviour and can be administered in a structured play session or using the board game version, depending on the age of the child. The BoHA takes $15 \mathrm{~min}$ to complete. The assessment is video-taped for later scoring by a rater blinded to group allocation and who has been certified in its use.

4. Self-care and mobility: PEDI-CAT: The PEDI-CAT is a standardised, norm-referenced assessment of indepen- 
dence in self-care. The test is valid, reliable and responsive in this population. ${ }^{19}$ The PEDI-CAT is completed by parents using an iPad or a computer application. The item bank of the PEDI-CAT was developed using Rasch measurement modelling on large samples of typically developing children and those with disabilities. Two domains, self-care and mobility, will be completed by caregivers.

5. Performance and satisfaction with occupational performance goals: The $\mathrm{COPM}^{20}$ will be used to measure performance of and satisfaction with individually defined selfcare, leisure or productivity goals. Test-retest reliability is high (ICC 0.76-0.89), and the COPM is responsive to change. ${ }^{20}$ Children 8 years and older can self-report, and caregivers can complete the COPM for younger children or those with cognitive difficulties that would preclude them from completing it independently. Children and their caregivers will set up to three goals. Perceived performance of an individualised goal and satisfaction with performance are rated on a 1-10 scale with higher scores reflecting higher perceived performance and satisfaction.

6. QOL: The CP QOL-Child is a 52-item, condition-specific self-report measure of child QOL that is specifically developed for measuring QOL in children with $\mathrm{CP}^{22}$ The majority of items have the stem 'How do you feel about...' with a response scale of 9 points from $1=$ very unhappy to $9=$ very happy. The domains covered in the child self-report version include physical well-being, social well-being, emotional well-being, school and acceptance by others. It has good concurrent validity, internal consistency (Cronbach's alpha 0.80-0.90) and testretest reliability for children 9 years of age and over. Significant discordance exists between child and parent proxy reports in many health-related QOL instruments, and both the child and parent proxy perspectives will be sought in the present study. The CP QOL will be completed by all children, unless they are under the age of 9 years or have an intellectual disability. An adult who is not participating in the study as the primary parent/caregiver will read the questionnaire alongside the child and clarify the meaning of the questions and response scale if necessary. For teenagers 13 years of age or older, the adolescent version will be completed (CP QOL-Teen) by teens and their caregiver. ${ }^{21}$

7. The CHU9D is a paediatric health-related QOL measure for use in economic evaluation. The measure consists of nine questions. Children can self-report from 7 years of age, and parents can proxy report for their child. In this study, the Child Health Utility 9D (CHU9D) will be completed by the child's primary caregiver. $^{44}$

\section{Data management}

Progress notes taken by treating therapists will be fully identified for legal reasons but will be stored confidentially in accordance with professional code of conduct and relevant legislation.
All other information will be coded with a participant ID number. Any identification codes will be stored in a different place from the data records to which they are linked. Data stored in electronic form will also be stored on the Queensland Cerebral Palsy and Rehabilitation Research Centre, The University of Queensland secure server with access limited to chief investigators and the study coordinator at the Queensland Cerebral Palsy and Rehabilitation Research Centre. Deidentified MRI data will be stored on a secure local server at the Australian E-Health Research Centre, CSIRO with access limited to chief investigators and named investigators on ethics. All consent forms and identifiable information will be stored in a separate, locked filing cabinet to the research data. Data management will comply with relevant privacy protocols, such as the Australian Standard on Personal Privacy Protection.

\section{Management of withdrawals}

Participants can withdraw at any time. Participants who choose to withdraw from the study will not be penalised in any way. If they wish to continue with therapy intervention for their child, they will be assisted to source another local therapy option that matches their preferences. Participants are informed of their right to withdraw at any time without consequences at the time of reading participant information forms and signing of consent forms. Participants can enrol and undergo HABIT-ILE irrespective of whether they consent to the neuroimaging and/ or economic analysis aspects of the study. Participants who withdraw will not be replaced, as the a priori power calculation will account for a $10 \%$ dropout rate and $10 \%$ crossover rate.

\section{Sample size estimation}

A 1.6 logit change on the ABILHAND-Kids was achieved in a small RCT of HABIT-ILE. ${ }^{14}$ A sample of 126 (63 in each arm) yields $80 \%$ power, with significance at a two-sided alpha level of 0.05 to show a difference of 1.6 ABILHAND-Kids logits, with an SD of change of 3 and buffering for $10 \%$ attrition. We will have $>90 \%$ power to detect a difference of 5 points or greater on the GMFM (assuming $\mathrm{SD}=6$ ) and alpha $=0.05$, buffering for $10 \%$ attrition). For neuroimaging outcomes, a $1 \%$ change in fractional anisotropy using fMRI-guided tractography and a $6 \%$ change in cortical thickness are considered realistic estimates for current therapies. Our recent work on power analysis for imaging measures of neuroplasticity in CP suggests that, assuming an $80 \%$ success rate of MRI, 39 subjects are required to detect a $1 \%$ change in FA using fMRI-guided tractography, the most sensitive available method. ${ }^{45}$

\section{Statistical analysis}

Analyses will follow standard principles for RCTs using two-group comparisons on all participants on an intention-to-treat basis. Primary comparison immediately postintervention (T2) and retention at (T3) based on 
ABILHAND-Kids and GMFM scores will be between treatment groups using linear regression with treatment group (HABIT-ILE/waitlist control) included as the main effect and baseline ABILHAND-Kids as the covariable. Effect estimates will be presented as mean difference and 95\% CIs. Secondary analyses will use similar methods to compare outcomes between groups immediately postintervention (T2) for brain structural integrity and structural connectivity (diffusion MRI-guided and fMRI-guided tractography), and at T2 and 26 weeks (T3) for clinical outcomes: walking distance, bimanual performance, self-care, mobility, performance of and satisfaction with individualised goals, and QOL. In cases where interval data are not able to be transformed appropriately for regression analyses, non-parametric methods (MannWhitney U) will be used for between-treatment comparisons. Sensitivity analyses of all outcomes will be conducted using multiple imputation techniques to investigate the effect of non-ignorable missing data during follow-up.

\section{Health economics}

A within-trial cost-utility analysis ${ }^{46}$ will be conducted to synthesise the costs and benefits of the HABIT-ILE programme compared with usual care. Resource use (staff/student time, equipment and facility use, and consumables) associated with the programme will be collected alongside the RCT. Healthcare use will be collected using a resource use questionnaire previously used in CP child studies ${ }^{47}$ Health utilities will be derived from the CHU9D, ${ }^{44}$ a generic child QOL measure designed specifically for economic evaluation, which has been validated in an Australian population. ${ }^{48}$ Incremental cost effectiveness ratios will be estimated and, where appropriate, sensitivity analyses will be undertaken.

\section{Ethics and dissemination}

Full ethical approval has been granted by the Children's Health Queensland Hospital and Health Service Human Research Ethics Committee (HREC/17/QRCH/282), the Medical Research Ethics Committee of The University of Queensland (2018000017/HREC/17/QRCH/2820) and Cerebral Palsy Alliance (2018_04_01/HREC/17/ QRCH/282). Participant information and consent forms will be provided to all participants and their caregivers prior to entering the study. Full written and informed consent will be obtained from all caregivers of children participating in the trial. The trial has been registered with the Australian and New Zealand Clinical Trial Registry. This protocol is reported according to the Standard Protocol Items: Recommendations for Intervention Trials statement ${ }^{49}$ and the TIDieR Checklist. ${ }^{24}$

Findings will be disseminated via peer-reviewed publication of study results, newsletter feedback to consumers, and presentation at key national and international conferences. The authors will plan a knowledge translation pathway if the intervention proves effective in improving ability to make and keep friends.

\section{Public/patient involvement statement}

Te patients and the public were not involved in the design or conduct of this study. Participants and their families will be informed of progress and outcomes of this study via newsletter and conferences open to consumers.

\section{DISCUSSION}

Over $60 \%$ of children with CP have bilateral motor impairment impacting independence in activities of daily living, participation and QOL. To date, there is limited evidence for effective interventions to improve motor and functional outcomes in children with bilateral CP. Building on a previous small study, ${ }^{14}$ the HABIT-ILE Australia project is the first large-scale study to test the efficacy of this intensive motor training intervention on motor and neuroimaging outcomes in children with bilateral CP. One potential limitation of the study is that therapy students under the supervision of trained therapists will be primarily delivering the HABIT-ILE intervention. This will be accounted for by providing 1 day of standardised training for all interventionists, daily debriefing meetings at the end of each day and ongoing daily feedback from supervising therapists. In addition, fidelity checks with the HABIT-ILE developer (YB) will occur throughout the conduct of each HABIT-ILE camp. Second, the dose being tested (65hours) was a pragmatic choice based on what is likely to be feasible and acceptable in the Australian context. This dose, however, is less than that in previous studies (90 hours). This study will additionally determine whether HABIT-ILE is translatable and implementable to a broad Australian setting.

The study has a number of strengths. The number of participants to be included has been calculated for both the primary clinical and secondary neuroimaging outcomes. Selected outcome measures have evidence for both validity and reliability in our population of interest. Development of standardised interventionist training and fidelity monitoring, in addition to a within-trial costutility analysis will provide vital information to inform the potential translation of this intervention. It is anticipated that results of this large RCT will be disseminated widely through peer-reviewed journals and academic conferences.

\section{Author affiliations}

${ }^{1}$ Queensland Cerebral Palsy and Rehabilitation Research Centre, The Faculty of Medicine, University of Queensland, South Brisbane, Queensland, Australia

${ }^{2}$ Institute of Neuroscience, Université Catholique de Louvain, Louvain-la-Neuve, Belgium

${ }^{3}$ Cerebral Palsy Alliance, Brookvale, New South Wales, Australia

${ }^{4}$ School of Occupational Therapy and Social Work, Curtin University, Perth, Western Australia, Australia

${ }^{5}$ CSIRO Health and Biosecurity, CSIRO Australian e-Health Research Centre, Herston, Queensland, Australia

${ }^{6}$ Faculty of Business, Economics and Law, University of Queensland, Wooloongabba, Queensland, Australia

${ }^{7}$ Menzies Health Institute Queensland, Griffith University, Nathan, Queensland, Australia 
Acknowledgements We acknowledge Mika Shimada and Natalie Dos Santos, study clinical research coordinators; Megan Thorley, occupational therapist, Meredith Wynter, physiotherapist, and Kate McLeod, physiotherapist, who are the clinical leads at the Brisbane site; Sherilyn Nolan, physiotherapist, who is the site coordinator for Perth; Bree Harmsen, occupational therapist, and Marissa Smith, physiotherapist, who are the clinical leads in Perth; Ashleigh Hines, occupational therapist, who is the site coordinator for Sydney; Anna te Velde, physiotherapist, and Emma Stanton, occupational therapist, are the clinical leads at the Sydney site; Julie Paradis, occupational therapist in Belgium for training and mentorship of Australian therapists in HABIT-ILE; Herston Imaging Facility, Brisbane, for performing MRIs at the Brisbane site; and I-Med New South Wales for performing the MRIs at the Sydney site.

Contributors LS was the chief investigator and, together with YB, RNB, IN, CE, CM, $\mathrm{KP}, \mathrm{DR}, \mathrm{PG}$ and RSW, designed, established and achieved funding for this study. LS, SR, IN and CM were responsible for ethics applications and reporting. LS, SR, $\mathrm{CM}, \mathrm{PG}, \mathrm{IN}$ and $\mathrm{CE}$ were responsible for recruitment and data collection. YB, LS and $S R$ were responsible for implementation of the interventionist training and fidelity monitoring. KP, JF and RB were responsible for the design and implementation of neuroimaging outcomes. LS, YB, RNB, IN, CE, CM and SR took the lead roles on the preparation for the publication of the clinical outcomes; KP, JF, LS, YB and RNB took the lead roles on the preparation on neuroscience publications; DR, LS and RNB took the lead roles for the preparation of health economic publications. RSW and MC provided biostatistical advice and oversight for all analyses and publications. LS and the chief investigators drafted the final version of this manuscript. All authors have contributed to the writing and critical review of the manuscript and have approved the final version. All data from this study will be submitted to peer review journals.

Funding This work is supported by the National Health and Medical Research Council (NHMRC) for a project grant (144846), NHMRC Career Development Fellowship (LS, 1160694) and NHMRC Research Fellowship (RNB, 1105038). Children's Hospital Foundation Mary McConnel Career Boost for Women (LS).

Competing interests None declared.

Patient consent for publication Not required.

Provenance and peer review Not commissioned; peer reviewed for ethical and funding approval prior to submission.

Data availability statement Data are available upon reasonable request.

Open access This is an open access article distributed in accordance with the Creative Commons Attribution Non Commercial (CC BY-NC 4.0) license, which permits others to distribute, remix, adapt, build upon this work non-commercially, and license their derivative works on different terms, provided the original work is properly cited, appropriate credit is given, any changes made indicated, and the use is non-commercial. See: http://creativecommons.org/licenses/by-nc/4.0/.

\section{REFERENCES}

1. ACPR Group. Australian cerebral palsy register report 2016, birth years 1993-2009. Sydney: Cerebral Palsy Alliance, 2016.

2. Galea C, Mcintyre S, Smithers-Sheedy H, et al. Cerebral palsy trends in Australia (1995-2009): a population-based observational study. Dev Med Child Neurol 2019;61:186-93.

3. Economics A. The economic impact of cerebral palsy in Australia in 2007. Canberra, ACT: Access Economics, 2008.

4. Novak I, Hines M, Goldsmith S, et al. Clinical prognostic messages from a systematic review on cerebral palsy. Pediatrics 2012;130:e1285-312.

5. Wake M, Salmon L, Reddihough D. Health status of Australian children with mild to severe cerebral palsy: cross-sectional survey using the child health questionnaire. Dev Med Child Neurol 2003;45:194-9.

6. Donkervoort M, Roebroeck M, Wiegerink D, et al. Determinants of functioning of adolescents and young adults with cerebral palsy. Disabil Rehabil 2007;29:453-63.

7. Michelsen SI, Uldall P, Kejs AMT, et al. Education and employment prospects in cerebral palsy. Dev Med Child Neurol 2005;47:511-7.

8. Sakzewski L, Ziviani J, Boyd RN. Efficacy of upper limb therapies for unilateral cerebral palsy: a meta-analysis. Pediatrics 2014;133:e175-204.

9. Novak I, Mclntyre S, Morgan C, et al. A systematic review of interventions for children with cerebral palsy: state of the evidence. Dev Med Child Neurol 2013;55:885-910.
10. Moreau NG, Bodkin AW, Bjornson K, et al. Effectiveness of rehabilitation interventions to improve gait speed in children with cerebral palsy: systematic review and meta-analysis. Phys Ther 2016;96:1938-54.

11. Chen Y-P, Pope S, Tyler D, et al. Effectiveness of constraint-induced movement therapy on upper-extremity function in children with cerebral palsy: a systematic review and meta-analysis of randomized controlled trials. Clin Rehabil 2014;28:939-53.

12. Bleyenheuft $\mathrm{Y}$, Arnould $\mathrm{C}$, Brandao MB, et al. Hand and arm bimanual intensive therapy including lower extremity (HABIT-ILE) in children with unilateral spastic cerebral palsy: a randomized trial. Neurorehabil Neural Repair 2015;29:645-57.

13. Saussez G, Brandão MB, Gordon AM, et al. Including a lowerextremity component during hand-arm bimanual intensive training does not attenuate improvements of the upper extremities: a retrospective study of randomized trials. Front Neurol 2017;8:495.

14. Bleyenheuft $Y$, Ebner-Karestinos D, Surana B, et al. Intensive upper- and lower-extremity training for children with bilatera cerebral palsy: a quasi-randomized trial. Dev Med Child Neurol 2017;59:625-33.

15. Plasschaert VFP, Vriezekolk JE, Aarts PBM, et al. Interventions to improve upper limb function for children with bilateral cerebral palsy: a systematic review. Dev Med Child Neurol 2019;61:899-907.

16. Reid LB, Boyd RN, Cunnington R, et al. Interpreting intervention induced neuroplasticity with $\mathrm{fMRI}$ : the case for multimodal imaging strategies. Neural Plast 2016;2016:1-13.

17. Maher CA, Williams MT, Olds TS. The six-minute walk test for children with cerebral palsy. Int J Rehabil Res 2008;31:185-8.

18. Elvrum A-KG, Zethræus B-M, Vik T, et al. Development and validation of the both hands assessment for children with bilateral cerebral palsy. Phys Occup Ther Pediatr 2018;38:113-26.

19. Haley SM, Coster WJ, Dumas HM, et al. Accuracy and precision of the pediatric evaluation of disability inventory computer-adaptive tests (PEDI-CAT). Dev Med Child Neurol 2011;53:1100-6.

20. Law M, Baptiste S, Carswell A, et al. Canadian occupational performance measure. 3rd edn. Ottawa,Ontario: CAOT Publications, 1998.

21. Davis E, Mackinnon A, Davern M, et al. Description and psychometric properties of the CP QOL-Teen: a quality of life questionnaire for adolescents with cerebral palsy. Res Dev Disabil 2013;34:344-52.

22. Waters E, Davis E, Mackinnon A, et al. Psychometric properties of the quality of life questionnaire for children with CP. Dev Med Child Neurol 2007;49:49-55.

23. Franki I, Van den Broeck C, De Cat J, et al. A study of whether video scoring is a reliable option for blinded scoring of the gross motor function Measure-88. Clin Rehabil 2015;29:809-15.

24. Hoffmann TC, Glasziou PP, Boutron I, et al. Better reporting of interventions: template for intervention description and replication (TIDieR) checklist and guide. BMJ 2014;348:g1687.

25. Sakzewski L, Ziviani J, Abbott DF, et al. Randomized trial of constraint-induced movement therapy and bimanual training on activity outcomes for children with congenital hemiplegia. Dev Med Child Neurol 2011;53:313-20.

26. Sakzewski L, Miller L, Ziviani J, et al. Randomized comparison trial of density and context of upper limb intensive group versus individualized occupational therapy for children with unilateral cerebral palsy. Dev Med Child Neurol 2015;57:539-47.

27. Sakzewski L, Provan K, Ziviani J, et al. Comparison of dosage of intensive upper limb therapy for children with unilateral cerebral palsy: how big should the therapy pill be? Res Dev Disabil 2015;37:9-16.

28. Bleyenheuft Y, Gordon AM. Hand-Arm bimanual intensive therapy including lower extremities (HABIT-ILE) for children with cerebral palsy. Phys Occup Ther Pediatr 2014;34:390-403.

29. Eliasson A-C, Krumlinde-Sundholm L, Rösblad B, et al. The manual ability classification system (MACS) for children with cerebral palsy: scale development and evidence of validity and reliability. Dev Med Child Neurol 2006;48:549-54.

30. Rosenbaum PL, Palisano RJ, Bartlett DJ, et al. Development of the gross motor function classification system for cerebral palsy. Dev Med Child Neurol 2008;50:249-53.

31. Palisano RJ, Rosenbaum P, Bartlett D, et al. Content validity of the expanded and revised gross motor function classification system. Dev Med Child Neurol 2008;50:744-50.

32. Hidecker MJC, Paneth N, Rosenbaum PL, et al. Developing and validating the communication function classification system for individuals with cerebral palsy. Dev Med Child Neurol 2011;53:704-10.

33. Mutlu A, Kara Özgün Kaya, Livanelioğlu A, et al. Agreement between parents and clinicians on the communication function levels and 
relationship of classification systems of children with cerebral palsy. Disabil Health J 2018;11:281-6.

34. Arnould C, Penta M, Renders A, et al. ABILHAND-Kids: a measure of manual ability in children with cerebral palsy. Neurology 2004;63:1045-52.

35. Bleyenheuft $\mathrm{Y}$, Gordon AM, Rameckers E, et al. Measuring changes of manual ability with ABILHAND-Kids following intensive training for children with unilateral cerebral palsy. Dev Med Child Neurol 2017:59:505-11.

36. de Jong LD, van Meeteren A, Emmelot $\mathrm{CH}$, et al. Reliability and sources of variation of the ABILHAND-Kids questionnaire in children with cerebral palsy. Disabil Rehabil 2018;40:684-9.

37. Elvrum A-KG, Saether R, Riphagen II, et al. Outcome measures evaluating hand function in children with bilateral cerebral palsy: a systematic review. Dev Med Child Neurol 2016;58:662-71.

38. Russell DJ, Avery LM, Rosenbaum PL, et al. Improved scaling of the gross motor function measure for children with cerebral palsy: evidence of reliability and validity. Phys Ther 2000;80:873-85.

39. Wang H-Y, Yang YH. Evaluating the responsiveness of 2 versions of the gross motor function measure for children with cerebral palsy. Arch Phys Med Rehabil 2006;87:51-6.

40. Wright FV, Boschen K, Jutai J. Exploring the comparative responsiveness of a core set of outcome measures in a schoolbased conductive education programme. Child Care Health Dev 2005;31:291-302.

41. Fiori S, Cioni G, Klingels K, et al. Reliability of a novel, semiquantitative scale for classification of structural brain magnetic resonance imaging in children with cerebral palsy. Dev Med Child Neurol 2014;56:839-45.
42. Reid LB, Cunnington R, Boyd RN, et al. Surface-Based fMRI-Driven diffusion tractography in the presence of significant brain pathology: a study linking structure and function in cerebral palsy. PLoS One 2016;11:e0159540.

43. Pagnozzi AM, Dowson N, Doecke J, et al. Identifying relevant biomarkers of brain injury from structural MRI: validation using automated approaches in children with unilateral cerebral palsy. PLoS One 2017;12:e0181605.

44. Stevens K. Valuation of the child health utility 9D index. Pharmacoeconomics 2012;30:729-47.

45. Reid LB, Pagnozzi AM, Fiori S, et al. Measuring neuroplasticity associated with cerebral palsy rehabilitation: an MRI based power analysis. Int J Dev Neurosci 2017.

46. Drummond M, Sculpher M, Claxton K, et al. Methods for economic evaluation of health care programs. New York, NY: Oxford University Press, 2015.

47. Boyd RN, Jordan R, Pareezer L, et al. Australian cerebral palsy child study: protocol of a prospective population based study of motor and brain development of preschool aged children with cerebral palsy. BMC Neurol 2013;13:57.

48. Ratcliffe J, Flynn T, Terlich F, et al. Developing adolescent-specific health state values for economic evaluation: an application of profile case best-worst scaling to the child health utility 9D. Pharmacoeconomics 2012;30:713-27.

49. Chan A-W, Tetzlaff JM, Altman DG, et al. Spirit 2013 statement: defining standard protocol items for clinical trials. Ann Intern Med 2013;158:200-7. 